\title{
Co-expression of KLK6 and KLKIO as prognostic factors for survival in pancreatic ductal adenocarcinoma
}

\section{F Rückert ${ }^{*, 1}$, M Hennig', CD Petraki', D Wehrum', M Distler', A Denz', M Schröder³, G Dawelbait ${ }^{3}$, H Kalthoff', H-D Saeger', EP Diamandis ${ }^{5}$, C Pilarsky ${ }^{1,6}$ and R Grützmann ${ }^{1,6}$}

'Visceral, Thoracic and Vascular Surgery, University Hospital Carl Gustav Carus, Technical University of Dresden, Fetscherstrasse 74, Dresden 01307, Germany; ' Department of Nephropathology, Evangelismos Hospital, Phedriadon 109, Athens I I 364, Greece; ${ }^{3}$ Bioinformatics Group, Biotechnological Centre, Technical University Dresden Tatzberg 47/49, Dresden 0 1307, Germany; ${ }^{4}$ Division of Molecular Oncology, Clinic for General Surgery and Thoracic Surgery, Schleswig-Holstein University Hospitals, Arnold-Heller-Str. 7, Kiel 24 105, Germany; ${ }^{5}$ Department of Pathology and Laboratory Medicine, Mount Sinai Hospital, 600 University Avenue, Toronto, ON M5G IX5, Canada

Kallikreins play an important role in tumour microenvironment and as cancer biomarkers in different cancer entities. Previous studies suggested an upregulation of KLKIO and KLK6 in pancreatic ductal adenocarcinoma (PDAC). Therefore, we evaluated the clinicopathological role of these kallikreins and their value as biomarkers in PDAC.

Differential expression was validated by DNA-microarrays and immunohistochemistry in normal and malignant pancreatic tissues. Sera concentrations of both kallikreins were evaluated using ELISA. In silico analysis of possible protein interactions and gene silencing of KLKIO in vitro using siRNAs gave further insights in the pathomechanisms.

Gene expression analysis and immunohistochemistry demonstrated a strong expression for KLKIO and KLK6 in PDAC. Statistical analysis showed that co-expression of these kallikreins correlated with an RI-resection status $(P=0.017)$ and worse outcome for overall survival $(P=0.03 \mathrm{I})$. Multivariate analysis proofed that co-expression is an independent prognostic factor for survival $(P=0.043)$. Importantly, KLKIO knockdown in AsPC-I cells significantly reduced cell migration, whereas computational analysis suggested interaction of $K L K 6$ with angiogenetic factors as an important mechanism.

Co-expression of KLKIO and KLK6 plays an unfavourable role in PDAC. Our results suggest that this effect is likely mediated by an interaction with the factors of the extracellular matrix and enhancement of cancer cell motility.

British Journal of Cancer (2008) 99, |484- |492. doi: I0.1038/sj.bjc.66047I7 www.bjcancer.com

Published online 14 October 2008

(C) 2008 Cancer Research UK

Keywords: pancreatic cancer; KLKIO; KLK6; DNA-microarray; microenvironment

Pancreatic ductal adenocarcinoma (PDAC) is one of the most aggressive cancers with an incidence rate of 6.3/100 000 (Lowenfels and Maisonneuve, 2006; Jemal et al, 2008). It is characterised by early metastasising and aggressive, infiltrative growth along the endothelium basement membrane and neurons (Eccles and Welch, 2007). This leaves more than $85 \%$ of the patients inoperable at the time of diagnosis. Unfortunately pancreatic carcinoma also shows an unsatisfactory response to oncological treatment (Wolff et al, 2000). This demonstrates the need for new therapeutic approaches and also for biomarkers, which make early diagnosis possible. Recently, we (Grutzmann et al, 2003) and others (IacobuzioDonahue et al, 2003; Yousef et al, 2004) showed that human kallikrein 10 and human kallikrein 6 are among the most highly and specifically overexpressed genes in pancreatic cancer compared with normal and benign pancreas tissues.

*Correspondence: Dr F Rückert, Visceral, Thoracic and Vascular Surgery, University Hospital Carl Gustav Carus, Technical University of Dresden, Haus 91, MTZ, Fetscherstr. 74, 1099 Dresden;

E-mail: Felix.Rueckert@uniklinikum-dresden.de

${ }^{6}$ These two authors shares the senior authorship.

Received 27 June 2008; revised 5 September 2008; accepted 17 September 2008; published online 14 October 2008
KLK10 and KLK6 are members of the kallikrein family of 15 known proteases in humans, which play an emerging role in tumour microenvironment, invasion and angiogenesis (Borgono and Diamandis, 2004). Kallikreins exert this function as secreted trypsin and chymotrypsin-like proteases by degradation of the extracellular matrix, which is an important reservoir for cytokines and growth factors such as VEGF, TGF- $\beta$ and kininogens (Borgono and Diamandis, 2004). Moreover, $K L K 3$, also known as prostatespecific antigen, is of great clinical value as a serological marker in prostate cancer (Borgono and Diamandis, 2004). Other members of the kallikrein family might also have a utility in screening of malignancies, like KLK11 and KLK6 for ovarian cancer (Diamandis et al, 2003; McIntosh et al, 2007). This leaves kallikreins as advantageous candidate genes for diagnosis and therapy in pancreatic cancer. Therefore, the aim of this study was to evaluate the clinicopathological role of these kallikreins and their value as biomarkers in PDAC.

$K L K 10$, also known as the normal epithelial cell-specific 1 is one of the newly identified members of the kallikrein family. Its role in carcinogenesis is unsolved as it is downregulated in some tumours such as breast cancer and acute lymphoblastic leukaemia, whereas overexpressed in ovarian, prostate or renal cancer (Luo et al, 2003; Petraki et al, 2003, 2006; Zhang et al, 2006). Unfortunately, the 
function of KLK10 protein remains poorly documented, neither the activators nor the substrates for $K L K 10$ are actually known (Zhang et al, 2006). KLK6, or protease $\mathrm{M}$, is highly expressed in several malignancies like ovarian, breast, colon or gastric cancer. It is correlated with lymphatic invasion and poor prognosis in gastric cancer (Yousef et al, 2004; Nagahara et al, 2005). KLK6 might exert this effect by the degradation of matrix proteins and thereby the augmentation of cancer cell motility and proliferation (Ghosh et al, 2004).

In this study, we show that kallikrein 10 and 6 demonstrate a strong protein expression in pancreatic carcinoma and are associated with poor patient prognosis and R1 resection status and thereby might contribute to the aggressive character of this malignancy.

The results indicate that this effect is most likely mediated by the interaction of KLK6 with factors of the extracellular matrix and the enhancement of cancer cell motility by KLK10.

\section{MATERIALS AND METHODS}

\section{Patients and demographic data}

For immunohistochemical analysis we used samples from 54 patients, operated from July 1996 until December 2003. None of the patients received adjuvant chemotherapy prior to operation. The eligibility criterion was a histologically confirmed PDAC. A positive microscopic resection margin (R1) was operationally defined as at least one cancer cell within $1 \mathrm{~mm}$ of any surface of the resected specimen. We included patients with the finding of metastases to the intra-aortocaval lymph nodes. Previous reports have shown that these metastases should not exclude patients in good condition from oncologic resection of the PDAC (Shrikhande et al, 2007). Relevant patient characteristics are summarised in Table 1.

For serum-ELISA several panels of sera were selected from 130 healthy donors, eight patients with benign tumours, 34 with inflammatory diseases and 28 patients with malignant diseases (Table 2). All sera were from patients treated at the University Hospital Dresden, Germany and University Hospital SchleswigHolstein, Germany. All patients had given informed consent.

\section{Construction of a virtual subarray and bioinformatic analysis}

For the construction of the virtual subarray we used data obtained from the U133 A/B Affymetrix GeneChip using extracted RNA from microdissected tissue as described earlier (Pilarsky et al, 2008).

The Cel Files obtained from the Affymetrix MAS 5.0 software were used for further analysis. The files were loaded into dCHIP 1.3 (www.dchip.org) then normalised, and expression values as well as absolute calls were calculated using the PM/MM model (Grutzmann et al, 2003). We scored genes as differentially expressed if they displayed a fold change $>2$. To identify signature genes we used the method described elsewhere (Grutzmann et al, 2004). Datasets are accessible (Arrayexpress E-MEXP-1121 and E-MEXP-950).

\section{Immunohistochemistry study and evaluation}

Immunostaining was carried out on paraffin-embedded tissue sections by streptavidin-biotin-peroxidase complex method using polyclonal antibodies for KLK6 and KLK10 (1:150). The ductal epithelium and the Langerhans' islets served as positive controls for both kallikreins (Petraki et al, 2001, 2002a, 2003). Negative controls were performed for all studied tissues by omitting the primary antibody (KLK6 and KLK10) or by replacing it by non-immune serum (dilution 1:500) (see Supplementary Data 1).

All sections were examined by one observer (CDP) blinded to both clinical and pathological data. Protein expression in PDACs was quantified using a visual grading system with a range between 0 and 2, based on the intensity and the proportion of positive tumour cells on the studied section: $0=$ no immunoexpression or weak staining in any proportion of the cancerous tissue, or moderate expression in $<I=5 \%$ of the cancerous tissue, $1=$ moderate staining in $5-50 \%$ of the cancerous tissue, $2=$ moderate staining in $>50 \%$ of the cancerous tissue or strong staining in any proportion of the cancerous tissue. According to staining intensity, cancers were classified as low, medium or high expressing tumours. Coexpression of both $K L K s$ was defined as strong if the sample showed strong expression for both $K L K \mathrm{~s}(2 / 2)$ and/or at least showed moderate expression for one $K L K(2 / 1)$. KLK6 and 10 immunoexpression were also screened in the normal pancreatic parenchyma (acinar, ductal and endocrine cells) and in the ampulla of Vater region of the small intestine.

\section{Patient serum selection and ELISA for kallikrein measurement in serum}

ELISA-type immunofluorometric procedures developed in-house were used to measure KLK6 and 10 levels in these sera. Assays used in this study were of the 'sandwich' type with the primary antibody used for capture and the secondary one for detection. Monoclonal-monoclonal combinations were used in this study. All ELISAs were tested negative for cross-reactivity against other kallikreins. Assay precision within the dynamic range was $<10 \%$. These assays were standardised with recombinant proteins produced in yeast or mammalian expression systems. More details about the kallikrein ELISA have recently been published (Shaw and Diamandis, 2007).

\section{Protein interaction prediction}

To evaluate the interactions we queried databases with known protein-protein interactions such as NetPro (www.molecular connections.com), SCOPPI (www.scoppi.org) and HPRD (www.hprd.org) and compared them to our data. To find possible novel interactions we used structure- and sequence-based prediction of protein interactions as described earlier (Altschul et al, 1997; Mishra et al, 2006; Dawelbait et al, 2007).

\section{Cell culture and transfection conditions}

The AsPC-1 cell line (ATCC Number CRL-1682), established from malignant ascites of a 62-year-old female caucasian, was used for this study. Cells were grown in RPMI-1640 (Invitrogen, Karlsruhe, Germany) with $2 \mathrm{~mm}$ L-glutamine, $1 \mathrm{~mm}$ sodium pyruvate, $4.5 \mathrm{gl}^{-1}$ glucose and $10 \%$ FCS in a humidified atmosphere containing $5 \%$ $\mathrm{CO}_{2}$ at $37^{\circ} \mathrm{C}$. AsPC- 1 cells $(50000)$ in media with $1 \%$ FCS were transfected with $600 \mathrm{ng}$ of siRNA using oligofectamine (Invitrogen $\mathrm{GmbH}$, Karlsruhe, Germany). Target sense sequences that effectively mediated silencing were as follows: KLK10.1 (UACAUG UCCUGGAUCAAUA) and KLK10.2 (UGACGUGCCUACCUCUU AG) (all from MWG Biotech, Ebersberg, Germany). Knockdown was confirmed by RT - PCR and western blot. siRNA against the green fluorescent protein (GGCUACGUCCAGGAGCGCACC) served as a negative control. For RT - PCR the following cell lines were used: Capan-1 (ATCC No. HTB-79), Capan-2 (ATCC No. HTB-80), MiaPaCa-2 (CRL-1420), Panc1 (ECACC No. 7092802), BXPC3 (CRL-1687) and Panc 89, Colo357, PancTUI, PT45P1 (all from Professor H Kalthoff, Kiel, Germany, (Sipos et al, 2003)). 
Table I Clinicopathological variables and KLKIO and KLK6 co-expression of the tumour cohort $(n=54)$

\begin{tabular}{|c|c|c|c|c|}
\hline \multirow[b]{2}{*}{ Characteristics } & \multirow{2}{*}{$\begin{array}{c}\text { All cases } \\
n(\%)\end{array}$} & \multicolumn{3}{|c|}{ Co-expression } \\
\hline & & None/low & Strong & $P$-value \\
\hline \multicolumn{5}{|c|}{ Age at diagnosis (years) 60.5 (range, $31-76)$} \\
\hline \multicolumn{5}{|l|}{ Gender } \\
\hline Male & $24(44.4)$ & 12 & 12 & 0.808 \\
\hline Female & $30(55.6)$ & 16 & 14 & \\
\hline \multicolumn{5}{|l|}{ Localisation } \\
\hline Head of pancreas & $47(87.0)$ & 26 & 21 & 0.186 \\
\hline Tail of pancreas & $7(13.0)$ & 2 & 5 & \\
\hline \multicolumn{5}{|l|}{ Tumour stage } \\
\hline pTI & $6(11.1)$ & 5 & I & 0.391 \\
\hline PT2 & $13(24.1)$ & 6 & 7 & \\
\hline PT3 & $32(59.3)$ & 16 & 16 & \\
\hline PT4 & $3(5.5)$ & । & 2 & \\
\hline \multicolumn{5}{|l|}{ Nodal status } \\
\hline NO & $22(40.7)$ & 12 & 10 & 0.565 \\
\hline $\mathrm{NI}$ & $31(57.4)$ & 15 & 16 & \\
\hline N2 & । (1.9) & 1 & 0 & \\
\hline \multicolumn{5}{|c|}{ Interaortocaval metastasis } \\
\hline Positive & $7(13.0)$ & 4 & 3 & 0.764 \\
\hline Negative & $47(87.0)$ & 24 & 23 & \\
\hline \multicolumn{5}{|l|}{ Grade } \\
\hline GI & $4(7.4)$ & 4 & 0 & 0.096 \\
\hline G2 & $38(70.4)$ & 17 & 22 & \\
\hline G3 & $12(22.2)$ & 7 & 5 & \\
\hline \multicolumn{5}{|l|}{ Residual tumour status } \\
\hline $\mathrm{R}_{0}$ & $41(75.9)$ & 25 & 16 & 0.017 \\
\hline $\mathrm{R}_{1}$ & $13(24.1)$ & 3 & 10 & \\
\hline \multicolumn{5}{|l|}{ Perineural invasion } \\
\hline Yes & $19(35.2)$ & 11 & 8 & 0.513 \\
\hline No & $35(64.8)$ & 17 & 18 & \\
\hline \multicolumn{5}{|l|}{ Perilymphatic invasion } \\
\hline Yes & $24(44.4)$ & 11 & 13 & 0.429 \\
\hline No & $30(55.6)$ & 17 & 13 & \\
\hline
\end{tabular}

Patients with strong immunohistochemical co-expression of KLK6 and KLKI 0 showed a significant correlation with $\mathrm{RI}$-Resection status $(P=0.017)$

Table 2 Results of the serum ELISA showed no statistical significance between diseases nor between different localisations

\begin{tabular}{|c|c|c|c|c|c|}
\hline \multirow{2}{*}{$\begin{array}{l}\text { Diagnosis } \\
\text { (Localisation) }\end{array}$} & \multicolumn{2}{|c|}{ Median } & \multicolumn{2}{|c|}{ Mean } & \multirow[b]{2}{*}{$P$ (log-rank) } \\
\hline & KLK6 & KLKIO & KLK6 & KLKIO & \\
\hline Benign Tumours $(n=4)$ & 12.4 & 2.1 & $10.3 \pm 7.8$ & $1.4 \pm 1.8$ & n.s. \\
\hline \multicolumn{6}{|l|}{ Inflammatory diseases } \\
\hline Bile duct $(n=1)$ & 12.9 & 1.3 & & & \\
\hline Gallbladder $(n=4)$ & 13.1 & 2.1 & $14 \pm 2.2$ & $2.3 \pm 0.67$ & \\
\hline Pancreas $(n=12)$ & 10 & 2.0 & $7.7 \pm 5.7$ & $2.4 \pm 0.79$ & \\
\hline \multicolumn{6}{|l|}{ Malignant tumours } \\
\hline Bile duct $(n=3)$ & 8.1 & 1.3 & $7.2 \pm 4.7$ & $1.2 \pm 0.31$ & \\
\hline Gallbladder $(n=3)$ & 5.7 & 1.0 & $4.5 \pm 2.0$ & $1.2 \pm 0.42$ & \\
\hline Pancreas $(n=8)$ & 9.7 & 1.6 & $9.3 \pm 4.6$ & $1.5 \pm 0.95$ & \\
\hline Normal $(n=65)$ & 9.5 & 1.8 & $9.0 \pm 3.6$ & $1.7 \pm 0.85$ & \\
\hline
\end{tabular}

\section{Reverse transcription-polymerase chain reaction}

Using RNeasy Mini Kit (Qiagen, Hilden, Germany) we isolated total RNA and subjected $500 \mathrm{ng}$ to cDNA synthesis using random primer and SuperScript II (Invitrogen GmbH, Karlsruhe, Germany). Of the synthesised cDNA $2 \%$ were used for quantitative RT-PCR. Analysis was performed using an ABI PRISM 5700 Sequence Detection System (Applied Biosystems, Weiterstadt, Germany). The genes were amplified with the Power SybrGreen PCR Master Mix according to the manufacturer's instructions. Gene expression was quantified by the comparative $C_{\mathrm{t}}$-Method, normalising $C_{\mathrm{t}}$-values to a housekeeping gene ( $\beta$-actin) and calculating the relative expression values. Each experiment was repeated two times in duplicate. The following primers were used: $\beta$-Actin: Actb 1498 f (AAGCCACCC-CACTTCTCTCTAA) and actb-1570R (AATGCTATCACCTCCCCTGTGT), KLK10: KLK10_f1 ex (GTCCTGGTGGACCAGAGTTG) and KLK10_r1_ex (GAGCT G-CTCTCCCTGAAGAA), KLK6: KLK6_f1 (GTG TGC TGG GGA TGA GAA GT) and KLK6_r1 (GGG ATG TTA CCC CAT GAC AC) (all from MWG Biotech, Ebersberg, Germany).

\section{Western blotting}

Cells were washed and lysed with LDS sample buffer (Invitrogen, Karlsruhe, Germany). Proteins were electrophoresed under reducing conditions on $4-12 \%$ acrylamide gels (Invitrogen, Karlsruhe, Germany) and then transferred to a nitrocellulose membrane (Hybond ECL, GE Healthcare, Munich, Germany). To block nonspecific binding, the membrane was incubated overnight in PBS with $0.1 \%$ Tween 20 (T-PBS) containing 5\% BSA at $4{ }^{\circ} \mathrm{C}$. Subsequently, the membrane was incubated for $1 \mathrm{~h}$ with the antibody against $K L K 10(1: 1000$, see (Petraki et al, 2001, 2002a, 2003)) or $\beta$-actin loading control $(1: 5000$, no. ab6276, Abcam, Cambridge, UK) in T-PBS and 5\% BSA. After washing in T-PBS, protein on the membrane was visualised using the ECL detection kit (GE Healthcare, Munich, Germany) with a peroxidase-labelled anti-mouse antibody for $\beta$-Actin $(1: 25000$, no. NIF 825 , Amersham Pharmacia, Amersham, United Kingdom) and peroxidase-labelled anti-rabbit antibody for $\operatorname{KLK10}(1: 5000$, no. NIF 824, Amersham Pharmacia, Amersham, United Kingdom) as per manufacturer's instructions. Protein expression was measured by AIDA evaluation software (Raytest, Straubenhardt, Germany) as the ratio of KLK10-staining intensity to actin-staining intensity.

\section{Boyden chamber assay}

Invasion in vitro was measured in Boyden chamber assay (no. 353097, BD Falcon, Heidelberg Germany). The PET membrane had a pore size of $8 \mu \mathrm{m}$ with a pore density of $1.0 \times 10^{5} \mathrm{~cm}^{-2}$. Cells were transfected using the above-mentioned protocol and incubated for $48 \mathrm{~h}$. Cells were then trypsinised, counted and cell suspensions of the two groups $\left(5 \times 10^{5}\right.$ cells per $\left.250 \mu \mathrm{l}\right)$ were transferred in $1 \%$ FCS medium onto the membrane. Then the chambers were put in 24-well plates containing 10\% FCS medium and cultured for $72 \mathrm{~h}$. Cells infiltrated through the reconstituted basement membrane and appeared on the outer surfaces. By HE staining the number of the cells was counted microscopically. Migration assays were repeated three times.

\section{Statistical analysis}

$P$-values were assigned using $\chi^{2}$ (Pearson) for the cross tables, the log-rank test (Mantel-Cox) for the survival univariate analysis by the Kaplan-Meier test, and the Cox regression analysis for multivariate survival analysis. For statistical analysis 'SPSS 13.0' for Windows was used. 
Table 3 (A): Results of the GeneChip analysis. The upregulated genes (fold-change $>2, P<5 \%$ ) are listed in the upper part, the downregulated genes in the lower part of the table; (B): Sequence-based and structure-based protein interaction prediction showed four possible interaction partners for KLK6

\begin{tabular}{|c|c|c|c|c|}
\hline Probe set & HGNC symbol & Fold change & $P$-value & \\
\hline \multicolumn{5}{|l|}{ 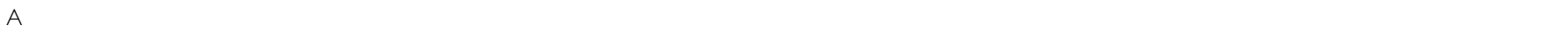 } \\
\hline \multicolumn{5}{|l|}{ Upregulated genes } \\
\hline KLKI0@215808_at & KLKIO & 1.6 & 0.01 & \\
\hline \multicolumn{5}{|l|}{ Downregulated genes } \\
\hline KLK13@216670_at & KLKI3 & -1.7 & 0.01 & \\
\hline KLKI2@234316_x_at & KLKI2 & -10.1 & $<0.01$ & \\
\hline KLKI@216699_s_at & KLKI & -10.2 & $<0.01$ & \\
\hline KLKI2@220782_x_at & KLKI2 & -10.5 & $<0.01$ & \\
\hline KLKI5@221462_x_at & KLKI5 & -15.3 & $<0.01$ & \\
\hline \multicolumn{5}{|l|}{$\mathrm{B}$} \\
\hline \multirow{2}{*}{$\begin{array}{l}\text { Protein I } \\
K L K 6\end{array}$} & Predicted partner & Also known as & Function & Compartment \\
\hline & SNCA & Synuclein & $\begin{array}{l}\text { Anti-apoptosis, central nervous system, } \\
\text { development, protein binding }\end{array}$ & Cytoplasm \\
\hline KLKIO & No interaction partner found & & & \\
\hline
\end{tabular}

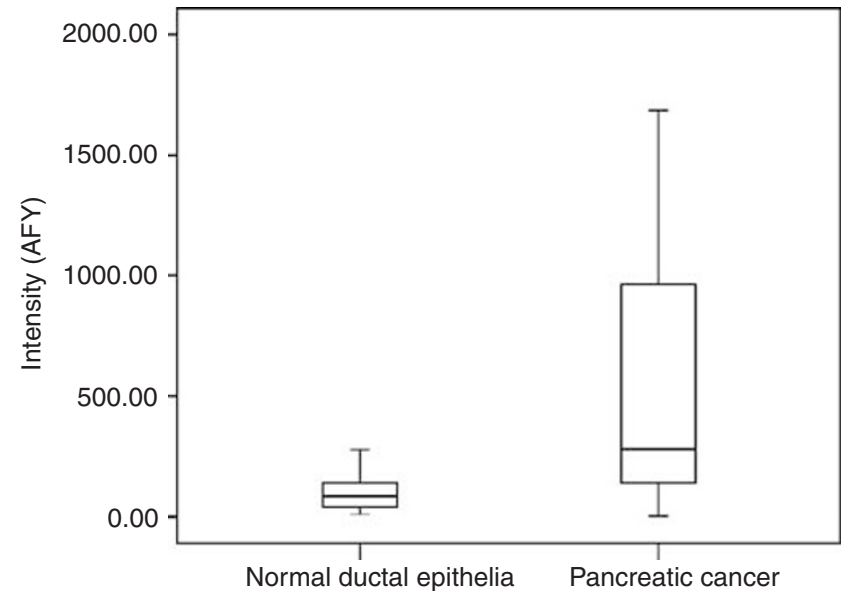

Figure I Results of the GeneChip analysis. KLKIO showed a marked upregulation in pancreatic cancer samples compared with normal individuals $(P=0.009)$.

\section{RESULTS}

\section{Virtual subarray}

Gene expression profiles of 19 patients with PDAC and normal ductal cells from 13 individuals were generated (Pilarsky et al, 2008). We then constructed a virtual subarray to identify gene expression changes of the 15 kallikreins. The nine probe sets identified with the virtual subarray analysis represented six differentially expressed genes (Table 3). Of these, KLK10 was overexpressed whereas $K L K 3,12,13$ and 15 were downregulated in PDAC compared with microdissected normal ductal cells. Upregulation of KLK10 was strong (Figure 1) compared with normal individuals $(P=0.009)$. We also decided to consider KLK6 for further evaluation because upregulation was found in PDAC by other groups (Iacobuzio-Donahue et al, 2003; Yousef et al, 2004).

\section{Immunohistochemical expression of KLK6 and KLK10}

In the endocrine pancreas, the immunohistochemistry for KLK6 and KLK10 showed strong staining in the endocrine cells of the Langerhans' islets and in scattered endocrine cells in connection with pancreatic ducts and acinar cells.

The exocrine part of the pancreas displayed a cytoplasmic expression in the small intercalated pancreatic ducts, the intraand inter-lobular pancreatic ducts, the main pancreatic duct and the common bile duct. Staining was absent in the acinar cells (Figure 2A and E). In the region of the ampulla of Vater in the small intestine, a strong cytoplasmic, mostly supranuclear immunoexpression was observed in the epithelium of the intestinal crypts. The absorptive cells in the surface villous epithelium showed a moderate cytoplasmic and brush border expression, whereas goblet cells were mostly negative (Figure $2 \mathrm{~B}$ ).

The staining for KLK6 in primary PDAC showed a moderate to strong expression in $91.5 \%$ of the cases, whereas it was only $64.4 \%$ for KLK10.

KLK6 showed a diffuse cytoplasmic immunostaining in the cancerous epithelium, whereas KLK10 mostly showed a patchy expression, often with luminal pattern (Figure 2). Analysis of immunohistochemistry revealed that patients with strong KLK6 and KLK10 co-expression had significant lower medium survival time of 20 months $(15.0-24.0)$ compared with patients without/ weak expression of these kallikreins (29 months (22.8-35.8)) $(P=0.031)$ (Figure 3). Neither KLK10 $(P=0.259)$ nor KLK6 $(P=0.452)$ expression could be correlated with survival alone. We could further associate high KLK6 and KLK10 immunoreactivity with R1-resection status $(P=0.017)$ (Table 1$)$.

Cox regression analysis identified $K L K 10$ and KLK6 co-expression as an independent prognostic factor with a statistically significant relationship to survival in PDAC $(P=0.043, \mathrm{RR} 2.002)$ (Table 4$)$. 

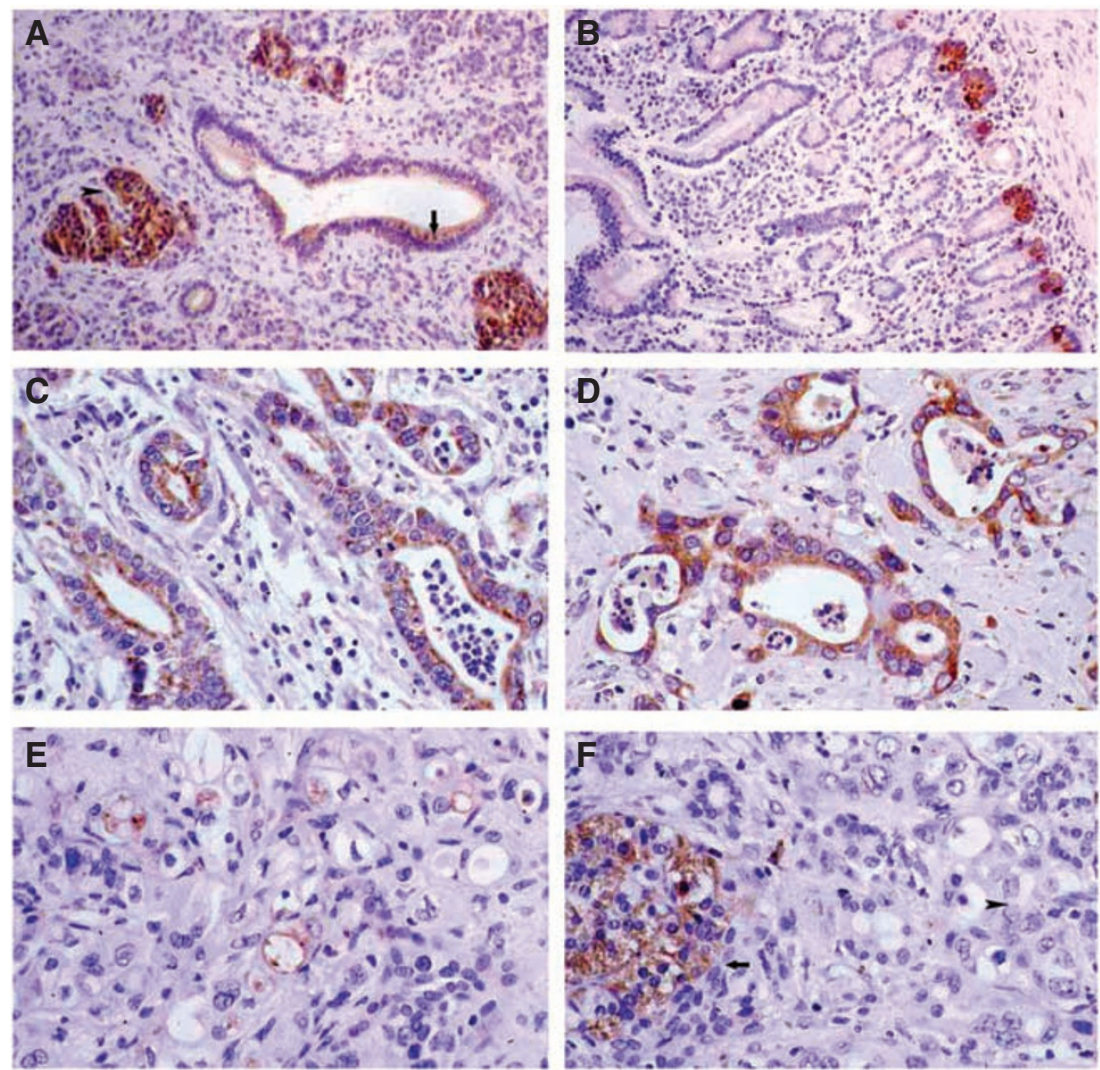

Figure 2 Immunohistochemical staining of PDAC samples. Moderate KLK6 immunoexpression in pancreatic ducts (arrow) and strong expression in Langerhans' islets (arrowhead) no staining in acini $(\times 100)(\mathbf{A})$. Strong KLK IO immunoexpression in the crypts of the intestinal epithelium of the ampulla of $\operatorname{Vater}(\times 100)(\mathbf{B})$. Strong KLK6 immunoexpression in pancreatic adenocarcinomas $(\times 200)(\mathbf{C}$ and $\mathbf{D})$. Moderate KLKI 0 immunoexpression in pancreatic adenocarcinomas $(\times 200)(\mathbf{E})$. Strong KLK10 immunoexpression in Langerhans' islets (arrow), absence of expression in pancreatic adenocarcinoma (arrowhead) $(\times 200)(\mathbf{F})$

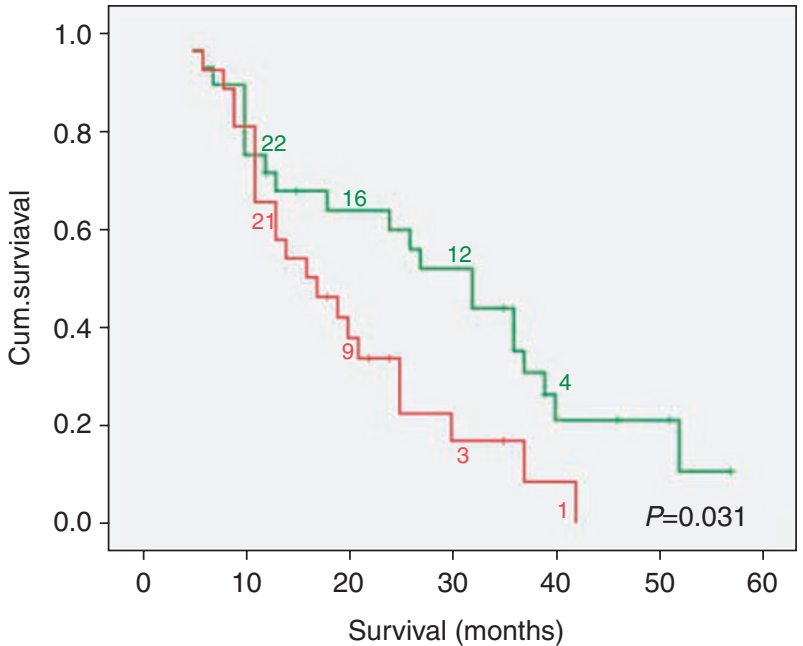

Figure 3 The survival curve shows a lower medium survival time of 20 months $(15.0-24.0)$ in the subgroup of patients with strong KLK6 and KLKIO co-expression compared with patients without/weak expression of these kallikreins (29 months $(22.8-35.8))(P=0.031)$.

\section{KLK10 and KLK6 serum concentration}

Using ELISA immunoassays developed in-house, we tested KLK6 and KLK10 serum levels in patients with malignant, inflammatory
Table 4 Cox regression model, including conventional variables and co-expression of KLK6 and KLKIO in all cases $(n=54)$

\begin{tabular}{lccc}
\hline & Relative risk & $\mathbf{9 5 \%} \mathbf{C l}$ & $\boldsymbol{P}$-value \\
\hline Co-expression KLK6/KLK10 & 2.022 & $1.021-4.006$ & 0.043 \\
Resection status & 0.571 & $0.247-1.321$ & 0.190 \\
Grading & 1.964 & $1.030-3.746$ & 0.040 \\
PT stage & 0.498 & $0.310-0.801$ & 0.004 \\
Nodal status & 1.814 & $0.937-3.513$ & 0.077 \\
Metastases & 0.545 & $0.177-1.679$ & 0.290 \\
\hline
\end{tabular}

Co-expression is a strong independent prognostic factor for survival in patients with $\operatorname{PDAC}(P=0.043)$

and benign diseases; additionally there was a panel of healthy patients.

There was no significant correlation of KLK6 and KLK10 serum levels with survival of the patients. Also, there was no statistical significance between healthy donors and patients with PDAC nor between different localisations of inflammatory, benign and malignant diseases of the pancreatico-biliary tract (Table 2).

\section{Protein interaction prediction}

To find potential interaction partners for KLK6 and 10, which might explain poor survival, we used two different computational methods: the structure- and the sequence-based protein interac- 
tion prediction. By means of these methods we could identify four potential interaction partners for KLK6: $\alpha-1$ antiproteinase, AT III, pigment epithelium-derived factor and synuclein (Table 3). However, no additional interaction partner for KLK10 other than the already described could be identified (Dawelbait et al, 2007).

\section{Expression of KLK10 and KLK6 in pancreatic cancer cell lines}

To evaluate expression of KLK10 and KLK6 in established cell lines we conducted a qRT - PCR. Capan-2, Panc89 and AsPC- 1 cell lines displayed the highest KLK10 expression. For KLK6 the cell lines Capan-2, Mia PaCa-2 and AsPC-1 showed a good expression. Like in immunohistochemistry of native tumour, KLK6 expression in cell lines was more distinct than that of KLK10 (Figure 4).

\section{Gene silencing of KLK10 in AsPC-1 cells}

To elucidate the relevance of dysregulations of KLK10 for carcinogenesis, we established a siRNA assay. For this purpose, we used the human pancreatic cancer cell line AsPC-1. This cell line showed detectable KLK10-expression as well as KLK6expression (Figure 4).

Cells were transfected with two KLK10 sequences, KLK10.1 and KLK10.2. Untreated cells and cells transfected with unspecific siRNA served as a negative control. As shown in Figure 5 the expression of $K L K 10$ was strongly inhibited compared with controls in RT-PCR $(P<0.001)$. The same holds true for protein synthesis in western blot $(P=0.025) . \beta$-actin levels showing equal quantities of protein were loaded (Figure 5); all experiments were
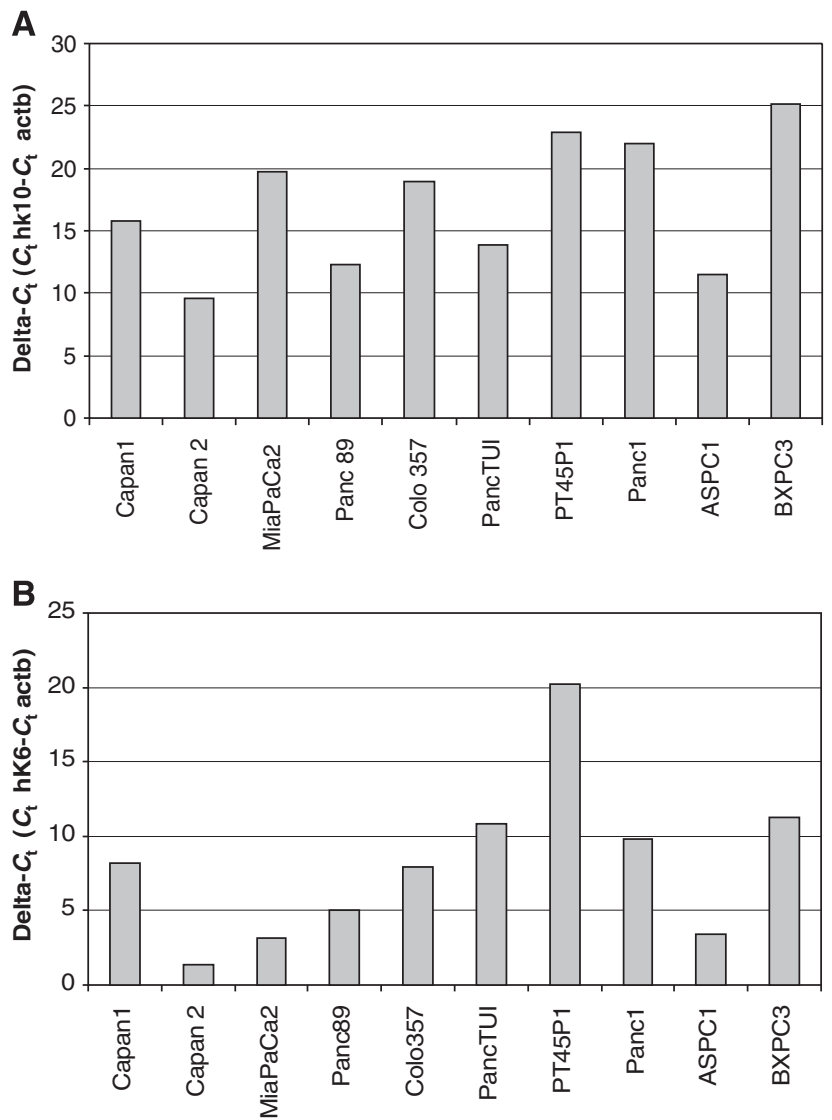

Figure 4 A qRT-PCR of different established pancreatic cancer cells lines showed Capan-2 and AsPC-I with relevant KLKIO-expression (A). KLK6-expression was high in nearly all measured cell lines (B). repeated four times. Because transfection with KLK10.1 showed the best inhibition of protein synthesis we chose this siRNA for our further studies. To examine off-target gene-silencing in our cells, we used the BLAST database (www.ncbi.nlm. nih.gov/BLAST) to search the human genome for any complementary sequences with at least 11 contiguous nucleotides matching the RNAi sites we used. No gene appeared to have sequence similarity. Transfection of AsPC-1 cells with KLK10.2 siRNA did not change the KLK6 expression levels as measured qRT - PCR (see Supplementary Data 1).

\section{Migration assay}

AsPC-1 cells transfected with KLK10.1 siRNA showed a significant decrease in cell motility compared with the GFP-transfected cells. Although only 636 cells $( \pm 285)$ could be isolated from the membrane in KLK10 knockout cells, it was $1189( \pm 508)$ in the control group $(P=0.05)$ (Figure 5$)$.

\section{DISCUSSION}

KLK10 and KLK6 are among the most highly and specifically overexpressed genes in pancreatic cancer compared with normal and benign pancreas tissues (Grutzmann et al, 2003; IacobuzioDonahue et al, 2003; Yousef et al, 2004).

Our study confirmed a marked overexpression of KLK10 in PDAC by means of a virtual subarray. Immunohistochemistry in native tumour tissue could prove not only an intense expression for $K L K 10$ in $64.4 \%$ of the malignant cells, but also for KLK6 in $91.5 \%$. Both proteins were located in the cytoplasm, from where they are likely to be secreted (Borgono et al, 2004).

Co-expression of different kallikreins, similar to the situation found in our study, was already reported in skin and different glands. In these tissues the kallikreins can act independently, but also together as part of proteolytic cascades (Petraki et al, 2002b; Borgono and Diamandis, 2004). The latter seems to be an important mechanism in pancreatic cancer, because expression of KLK10 itself could not be associated with poor survival in PDAC, whereas the co-expression of both kallikreins was significantly associated with poor survival and an R1-resection status, which is an indirect sign for infiltrative and aggressive growth. In multivariate analysis, the co-expression of KLK10 and KLK6 was also an independent risk factor for survival.

It is most interesting, in which ways kallikreins affect cellular signalling and thereby contribute to cancer progression. It was already reported that kallikreins influence communication between malignant cells and their environment by degradation of extracellular matrix and thereby facilitate tumour invasion and metastasis (Borgono and Diamandis, 2004). In case of $K L K 6$, degradation of fibrinogen, laminin, fibronectin and collagen types I and IV are documented (Bernett et al, 2002; Magklara et al, 2003). This cleavage of fractions of the ECM might be of specific importance in pancreatic carcinoma, which is a tumour type with a very high content of stromal tissue (Pilarsky et al, 2008). In contrast, functional data on KLK10 are very limited. Although Zhang et al (2006) suggested, that KLK10 was not even an active protease, it was stated in the same report that neither the protein relevant for conversion of $K L K 10$ into its active form nor the physiological substrates for KLK10 are known. So, the importance of KLK10 in tumour progression remains unclear.

It therefore seems crucial to further pinpoint some of the components, which might be responsible for the pathophysiological effect of KLK10. To find possible interaction partners for both kallikreins we used the in silico method of protein interaction prediction. By means of this method we could identify four potential interaction partners for KLK6. Although $\alpha-1$ antiproteinase seems to be an inhibitor for KLK6 action, the interaction with 
A
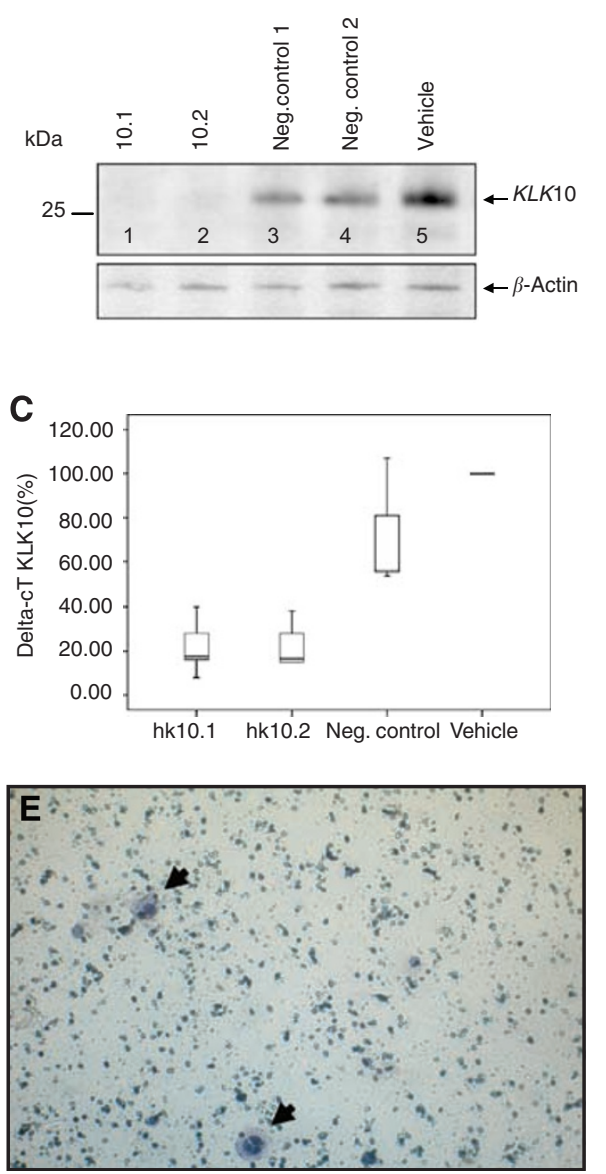

B
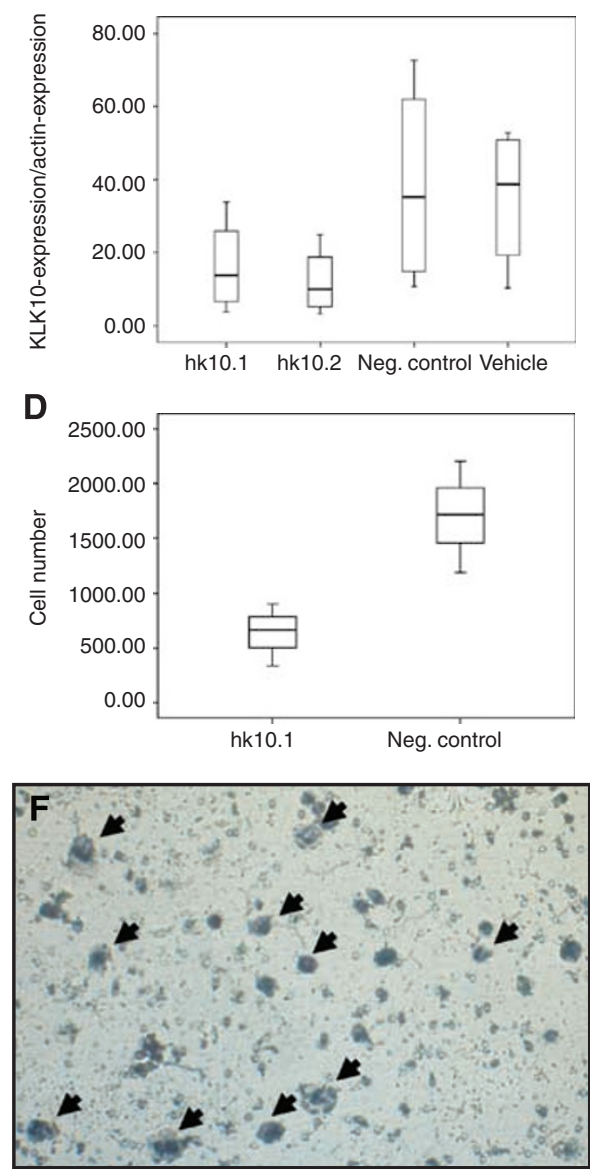

Figure 5 AsPC-I cells were transfected with two KLKIO-sequences, KLKIO.I and KLKI0.2. Transfection resulted in a strong downregulation of KLKIO protein synthesis in the western blot. Cell lysates of KLKI0.I - and KLKI0.2-transfected cells showed nearly no staining (lanes I and 2) compared with the controls (lanes 3-5) (A). Statistical analysis showed a statistical downregulation of the transfected group compared with the control group $(P=0.025)(\mathbf{B})$. The same holds true for gene expression in RT-PCR $(P<0.00$ I) $(\mathbf{C})$. The Boyden chamber migration assay: Invasion in vitro was measured as described in 'Materials and methods'. Statistical analysis showed that KLKIO.I-transfected cells had a significant decrease in cell motility compared with the controls $(P=0.05)(\times 200)(\mathbf{D})$. Only few KLKI O.I-transfected AsPC-I cells migrated through the membrane (arrow) (E). The control group displayed normal cell migration (arrows) (F).

AT III shows a branching between kallikreins and blood coagulation cascade, as already reported earlier (Borgono et al, 2004). Another interaction partner was pigment epitheliumderived factor (PEDF), which is the major circulating inhibitor of plasmin. With this interaction, PEDF is linked to the plasminogen activator/plasmin system, which is one of the main protease systems involved in tumour cell invasion and metastasis (Hayashido et al, 2007). Only recently PEDF was also identified as a key inhibitor of stromal vasculature in the mural pancreas. In vivo androgen ablation increased PEDF in human cancer biopsies, which might also be an indirect sign for the interaction of the androgen-responsive kallikrein family and PEDF (Doll et al, 2003). The interaction between KLK6 and PEDF seems highly significant and studies are under way which will further evaluate this topic. Another interaction partner we found is synuclein, which integrates presynaptic signalling and membrane trafficking in neurons. The high expression of KLK6 might thereby play an important role in various pathologic processes of pancreatic cancer.

Although a specific interaction partner for KLK10 could not be found, our study implies that it might have a role in the pathophysiology of PDAC. To ascertain the contribution of KLK10 to pancreatic cancer microenvironment, we used siRNA- mediated gene-silencing (Hammond et al, 2000). AsPC-1 cells, which inherently express high levels of KLK10 mRNA, were transfected with specific siRNA. We could not observe an effect on proliferation or apoptosis in KLK10-silenced cells (data not shown). But KLK10-suppressed clones had markedly reduced cell motility in the Boyden chamber assay. The number of cells migrating through the membrane along an FCS-gradient dropped more than $50 \%$. This is highly significant, as KLK6 was also shown to reduce cell motility (Ghosh et al, 2004).

Although high expression in pancreatic carcinoma indicates that KLK6 and 10 could be promising tumour markers, we could not assess the use of KLK6 and KLK10 as serum biomarkers in PDAC. The serum levels of both proteins showed no significant differences between patients with PDAC and healthy donors. In addition, the serum concentrations were not able to predict the localisation of malignant lesions in the pancreatico-biliary tract. This circumstance can be because of the mainly local action of the kallikreins or fast degradation. Probably future studies including more patients can prove a use for KLK6 or KLK10 as tumour biomarkers in PDAC.

In conclusion, this study shows that KLK10 and KLK6 co-expression has an unfavourable influence on the survival in patients with PDAC and was significantly associated with R1 
resection status. This effect might be mediated by direct or indirect interaction of the two kallikreins. The pathophysiological mechanisms are most likely degradation of the extracellular matrix and interaction with angiogenic factors by KLK6, whereas $K L K 10$ augments cell motility. However, our findings suggest a high complexity of interactions between the kallikreins, which leaves it difficult to generally make statements about properties of single kallikreins.

It seems very promising to find out more about the physiological role of KLK10. Consequently, it might be possible to use inhibitors of kallikreins to disrupt interactions between the tumour and its environment and thereby reduce disease progression in patients with pancreatic cancer.

\section{ACKNOWLEDGEMENTS}

We thank Ms Beatrix Jahnke and Mrs Anne Lehner for technical support.

Supplementary Information accompanies the paper on British Journal of Cancer website (http://www.nature.com/bjc)

\section{REFERENCES}

Altschul SF, Madden TL, Schaffer AA, Zhang J, Zhang Z, Miller W, Lipman DJ (1997) Gapped BLAST and PSI-BLAST: a new generation of protein database search programs. Nucleic Acids Res 25: 3389-3402

Bernett MJ, Blaber SI, Scarisbrick IA, Dhanarajan P, Thompson SM, Blaber M (2002) Crystal structure and biochemical characterization of human kallikrein 6 reveals that a trypsin-like kallikrein is expressed in the central nervous system. J Biol Chem 277: 24562-24570

Borgono CA, Diamandis EP (2004) The emerging roles of human tissue kallikreins in cancer. Nat Rev Cancer 4: 876-890

Borgono CA, Michael IP, Diamandis EP (2004) Human tissue kallikreins: physiologic roles and applications in cancer. Mol Cancer Res 2: 257-280

Dawelbait G, Winter C, Zhang Y, Pilarsky C, Grutzmann R, Heinrich JC, Schroeder M (2007) Structural templates predict novel protein interactions and targets from pancreas tumour gene expression data. Bioinformatics 23: $\mathrm{i} 115$ - i124

Diamandis EP, Scorilas A, Fracchioli S, Van Gramberen M, De Bruijn H, Henrik A, Soosaipillai A, Grass L, Yousef GM, Stenman UH, Massobrio M, Van Der Zee AG, Vergote I, Katsaros D (2003) Human kallikrein 6 (hK6): a new potential serum biomarker for diagnosis and prognosis of ovarian carcinoma. J Clin Oncol 21: $1035-1043$

Doll JA, Stellmach VM, Bouck NP, Bergh AR, Lee C, Abramson LP, Cornwell ML, Pins MR, Borensztajn J, Crawford SE (2003) Pigment epithelium-derived factor regulates the vasculature and mass of the prostate and pancreas. Nat Med 9: 774-780

Eccles SA, Welch DR (2007) Metastasis: recent discoveries and novel treatment strategies. Lancet 369: $1742-1757$

Ghosh MC, Grass L, Soosaipillai A, Sotiropoulou G, Diamandis EP (2004) Human kallikrein 6 degrades extracellular matrix proteins and may enhance the metastatic potential of tumour cells. Tumour Biol 25: $193-199$

Grutzmann R, Foerder M, Alldinger I, Staub E, Brummendorf T, Ropcke S, Li X, Kristiansen G, Jesnowski R, Sipos B, Lohr M, Luttges J, Ockert D, Kloppel G, Saeger HD, Pilarsky C (2003) Gene expression profiles of microdissected pancreatic ductal adenocarcinoma. Virchows Arch 443: $508-517$

Grutzmann R, Pilarsky C, Ammerpohl O, Luttges J, Bohme A, Sipos B, Foerder M, Alldinger I, Jahnke B, Schackert HK, Kalthoff H, Kremer B, Kloppel G, Saeger HD (2004) Gene expression profiling of microdissected pancreatic ductal carcinomas using high-density DNA microarrays. Neoplasia 6: 611-622

Hammond SM, Bernstein E, Beach D, Hannon GJ (2000) An RNA-directed nuclease mediates post-transcriptional gene silencing in Drosophila cells. Nature 404: $293-296$

Hayashido Y, Hamana T, Ishida Y, Shintani T, Koizumi K, Okamoto T (2007) Induction of alpha2-antiplasmin inhibits E-cadherin processing mediated by the plasminogen activator/plasmin system, leading to suppression of progression of oral squamous cell carcinoma via upregulation of cell-cell adhesion. Oncol Rep 17: 417-423

Iacobuzio-Donahue CA, Ashfaq R, Maitra A, Adsay NV, Shen-Ong GL, Berg K, Hollingsworth MA, Cameron JL, Yeo CJ, Kern SE, Goggins M, Hruban RH (2003) Highly expressed genes in pancreatic ductal adenocarcinomas: a comprehensive characterization and comparison of the transcription profiles obtained from three major technologies. Cancer Res 63: 8614-8622

Jemal A, Siegel R, Ward E, Hao Y, Xu J, Murray T, Thun MJ (2008) Cancer statistics, 2008. CA Cancer J Clin 58: 71-96
Lowenfels AB, Maisonneuve P (2006) Epidemiology and risk factors for pancreatic cancer. Best Pract Res Clin Gastroenterol 20: 197-209

Luo LY, Katsaros D, Scorilas A, Fracchioli S, Bellino R, van Gramberen M, de Bruijn H, Henrik A, Stenman UH, Massobrio M, van der Zee AG, Vergote I, Diamandis EP (2003) The serum concentration of human kallikrein 10 represents a novel biomarker for ovarian cancer diagnosis and prognosis. Cancer Res 63: $807-811$

Magklara A, Mellati AA, Wasney GA, Little SP, Sotiropoulou G, Becker GW, Diamandis EP (2003) Characterization of the enzymatic activity of human kallikrein 6: autoactivation, substrate specificity, and regulation by inhibitors. Biochem Biophys Res Commun 307: $948-955$

McIntosh MW, Liu Y, Drescher C, Urban N, Diamandis EP (2007) Validation and characterization of human kallikrein 11 as a serum marker for diagnosis of ovarian carcinoma. Clin Cancer Res 13: $4422-4428$

Mishra GR, Suresh M, Kumaran K, Kannabiran N, Suresh S, Bala P, Shivakumar K, Anuradha N, Reddy R, Raghavan TM, Menon S, Hanumanthu G, Gupta M, Upendran S, Gupta S, Mahesh M, Jacob B, Mathew P, Chatterjee P, Arun KS, Sharma S, Chandrika KN, Deshpande N, Palvankar K, Raghavnath R, Krishnakanth R, Karathia H, Rekha B, Nayak R, Vishnupriya G, Kumar HG, Nagini M, Kumar GS, Jose R, Deepthi P, Mohan SS, Gandhi TK, Harsha HC, Deshpande KS, Sarker M, Prasad TS, Pandey A (2006) Human protein reference database-2006 update. Nucleic Acids Res 34: D411-D414

Nagahara H, Mimori K, Utsunomiya T, Barnard GF, Ohira M, Hirakawa K, Mori M (2005) Clinicopathologic and biological significance of kallikrein 6 overexpression in human gastric cancer. Clin Cancer Res 11: 6800-6806

Petraki CD, Gregorakis AK, Papanastasiou PA, Karavana VN, Luo LY, Diamandis EP (2003) Immunohistochemical localization of human kallikreins 6, 10 and 13 in benign and malignant prostatic tissues. Prostate Cancer Prostatic Dis 6: 223-227

Petraki CD, Gregorakis AK, Vaslamatzis MM, Papanastasiou PA, Yousef GM, Levesque MA, Diamandis EP (2006) Prognostic implications of the immunohistochemical expression of human kallikreins 5, 6, 10 and 11 in renal cell carcinoma. Tumour Biol 27: 1-7

Petraki CD, Karavana VN, Luo LY, Diamandis EP (2002a) Human kallikrein 10 expression in normal tissues by immunohistochemistry. J Histochem Cytochem 50: 1247-1261

Petraki CD, Karavana VN, Revelos KI, Luo LY, Diamandis EP (2002b) Immunohistochemical localization of human kallikreins 6 and 10 in pancreatic islets. Histochem J 34: $313-322$

Petraki CD, Karavana VN, Skoufogiannis PT, Little SP, Howarth DJ, Yousef GM, Diamandis EP (2001) The spectrum of human kallikrein 6 (zyme/ protease $\mathrm{M} /$ neurosin) expression in human tissues as assessed by immunohistochemistry. J Histochem Cytochem 49: 1431 - 1441

Pilarsky C, Ammerpohl O, Sipos B, Dahl E, Hartmann A, Wellmann A, Braunschweig T, Lohr M, Jesnowski R, Friess H, Wente MN, Kristiansen G, Jahnke B, Denz A, Ruckert F, Schackert HK, Kloppel G, Kalthoff H, Saeger HD, Grutzmann R (2008) Activation of Wnt signalling in stroma from pancreatic cancer identified by gene expression profiling. J Cell Mol Med [e-pub ahead of print]

Shaw JL, Diamandis EP (2007) Distribution of 15 human kallikreins in tissues and biological fluids. Clin Chem 53: 1423-1432

Shrikhande SV, Kleeff J, Reiser C, Weitz J, Hinz U, Esposito I, Schmidt J, Friess H, Buchler MW (2007) Pancreatic resection for M1 pancreatic ductal adenocarcinoma. Ann Surg Oncol 14: 118-127 
Sipos B, Moser S, Kalthoff H, Torok V, Lohr M, Kloppel G (2003) A comprehensive characterization of pancreatic ductal carcinoma cell lines: towards the establishment of an in vitro research platform. Virchows Arch 442: 444-452

Wolff RA, Chiao P, Lenzi R, Pisters PW, Lee JE, Janjan NA, Crane $\mathrm{CH}$, Evans DB, Abbruzzese JL (2000) Current approaches and future strategies for pancreatic carcinoma. Invest New Drugs 18: 43-56

Yousef GM, Borgono CA, Popalis C, Yacoub GM, Polymeris ME, Soosaipillai A, Diamandis EP (2004) In-silico analysis of kallikrein gene expression in pancreatic and colon cancers. Anticancer Res 24: $43-51$

Zhang Y, Bhat I, Zeng M, Jayal G, Wazer DE, Band H, Band V (2006) Human kallikrein 10, a predictive marker for breast cancer. Biol Chem 387: $715-721$ 